\title{
Talea: An Extensible Framework for E-Business Integration
}

\author{
G. Levi, A. Vagliengo and A. Goy
}

\section{Introduction and Motivating Scenario}

This paper describes Talea [12], a platform aimed at supporting the development of web-based e-business applications. Talea is a software designed and developed within Diadi 2000 [4], a Piedmont Region project co-founded within the European Structural Funds framework. The main goal of Diadi 2000 is to support the technology transfer to SME (SME) in the Piedmont Region, in order to increase their competitiveness and technological development.

We will briefly describe the context in which the Diadi 2000 project has been conceived and carried on, since it is very important to understand the motivations underlying Talea architecture and functionality. Italian SME, especially in the north-west area (Piedmont), are traditionally reluctant to the introduction of technological innovation. Their business strategies are usually based on wellestablished practices, in which face-to-face interaction plays a major role and new technologies, or new business models, are often considered with suspicion and distrust. For example, the majority of SME currently have a web site, but a very small number of these sites are actually integrated with their business activities (transaction management, customer care, marketing, etc.)1.

In this perspective, the Enterprise Application Integration process [1], [15] must start from a change in mental attitudes towards innovation and thus the main goal of the Diadi 2000 project is to convince SME that the exploitation of ICT technologies could represent a real added value to their business. In particular, the project, by means of the Talea software platform, aims at introducing Semantic Web technologies, and in particular shared and customizable ontologies, in

According to the report MATE-Commercio Elettronico Italia, "Osservatorio PMI I semestre 2003” (www.commercenet.it), in 2003 almost all Italian PMI (small/medium enterprises) had an Internet connection, two out of three of them had a static web site, but only 5,5\% exploited the Internet to do business on-line. 
business processes in order to support the customer-supplier interaction, in an Enterprise Application Integration perspective.

Talea can be viewed as a generic matchmaker for e-business (see [13], [14]), since it supports a flexible matching between service provision and request: it enables service providers to advertise their products and services, and customers to search for resources they are looking for2. Talea was conceived and designed in order to facilitate business improvement for SME, interested in adopting ICT without eccessive starting costs. As it has been claimed in many projects (see, for instance, [5], [3], [8]), the introduction of a semantic matchmaker in a serviceoriented architecture (SOA) has the goal of enhancing the capabilities of a typical e-business framework. In particular, it can represent an improvement of SOA, since it adds more powerful description and classification mechanisms. See also [13] and [7], who provide an effective overview of the application of Semantic Web technologies to SOA and e-commerce systems. This perspective represents the framework that supported the design and development of the first Talea prototype that was conceived with the main goal of being easily used by SME after a simple customization process. For instance, a small family-based wineproduction enterprise can increase its productivity by exploiting Talea to advertise its products, possibly joining the row process as a customer, with respect to grape suppliers, and as a provider, with respect to wine customers.

The main obstacle to be faced in order to achieve this goal is, again, a change in the mental attitude of SME leaders: the Diadi 2000 project, by means of Talea, aims at showing that a different approach, based on Semantic Web standards coupled with an Object-Orientd architecture and some Aspect-Oriented architectural adds, can actually result in an improvement of the customer-supplier interaction and in a more tight integration of enterprise systems. These results, in turn, can increase the effectiveness of business management, and thus can represent a competitive advantage. Within the described scenario, the design of Talea had to represent a trade-off between innovation and acceptance: the system had to show effective but simple features, in order to have a chance to be accepted. Moreover, this scenario imposed two furher important requirements: (i) Easy customization, essential for the instantiation of Talea in different domains, i.e., for its exploitation by a significant number of SME, with a relatively small effort. (ii) Multi-device acces, needed since both customers and suppliers rely on wireless connections and mobile devices (PDA, smartphones, etc.) to buy/offer services.

In order to provide a first trial, Talea was exploited to test a prototype service supporting the collaboration process between minor actors of the local tourist sector and the Local Tourist Organization Agency. The service was tested in the mountain area that will host in Piedmont the next Winter Olympic Games in February 2006, with the support of the Local Tourist Agency MontagneDoc.

After this testing phase (June-September 2005), Talea has been released in compliance with the general principles of Open Source and its licences, with the

2 The current prototype does not support automatic negotiation: when customers reserve products/services, Talea simply notifies the corresponding providers about such a reservation (by e-mail, sms, or fax). 
aim of encouraging its use to implement and provide high quality personalized services within the supply chain. Talea has been made available for small and medium enterprises with the aim of increasing their local competitiveness, introducing ICT within cooperation processes and transferring towards local companies, belonging to the computer science and ICT sectors, a new model of reuse and customization of basic software components. In line with this aim, a first group of local companies has been selected in order to apply Talea technology in their business. The project called "A network for Small Local Trade" is one of the eight DIADI 2000 Pilot Projects, developed in a social disadvantaged area in the city of Turin. In particular, Biloba Cooperative Society, as implementer and responsible for the Pilot Project, has pointed out the need of local dealers for an innovative solution to increase their competitiveness. The technological innovation of the platform regards: (i) The integration of Talea within an e-commerce portal that could be updated through mobile phones and Digital Terrestrial Television. The Pilot Project, exploiting the potentialities offered by Talea, provides users with an easy access to new technologies, using familiar devices. (ii) The development of a GIS module and in particular a geographic interface and a routes optimization system to be integrated in Talea framework.

\section{What is Talea}

The exploitation of Talea in order to implement e-business applications requires the customization of the framework to specific domains. In the following we will refer to the person (or the team) who performs such a customization as the customizer. The result of the customization is an application that can be exploited by the final user (from now on, simply, user) to provide and consume resources (products or services). Talea users can be both providers and consumers3. Provider users are SME, while customer users can be individuals, agencies or enterprises. For instance, in the first prototype, the domain is mountain tourism; customer users are local travel agencies and provider users are mountain guides, innkeepers, etc.

The design of the Talea framework has been focused on the customizer, in order to support customization at three different levels: the presentation layer, the business logic, and the application domain. Such a customization is supported by three main mechanisms:

- an infrastructure that supports the matching between provider and consumer (Talea Backend);

- a customization mechanism that supports the description of the particular application domain (Talea Ontology extension);

- a programming pattern that supports the easy implementation of new features, to extend functionality (Generator/Performer Pattern).

\footnotetext{
3 Sometimes it is the case that a single entity plays a double role, acting both as provider
} and consumer. 
The customization of the presentation layer requires the design of the final user interface, handled by the Frontend and providing multi-device access (see Sect. 3); the customization of the business logic involves the implementation of new features to extend the functionality of the Talea Backend (see Sect. 3), while the customization of the knowledge about the domain is achieved through the extension of the the Talea Ontology (see Sect. 4).

\section{Architecture}

Talea architecture (see Fig. 1) was designed in order to facilitate the integration of different business processes, with particular attention to make domain-specific customization easy. This principle suggested us a number of criteria for the design of the system architecture, i.e.: the split of the system in two main separated parts, i.e. a Backend, containing the business logic, and a Frontend, managing the presentation layer; the implementation of principles of service-oriented applications (i.e. XML-based communication an representation); the exploitation of specific design patterns that guarantee a high modularity.

As far as the principles of typical service-oriented applications are concerned, the architecture relies on XML messages to communicate data among system modules, and on XML-based standards (e.g. RDFS) to represents available resources, in order to support browsing and search.

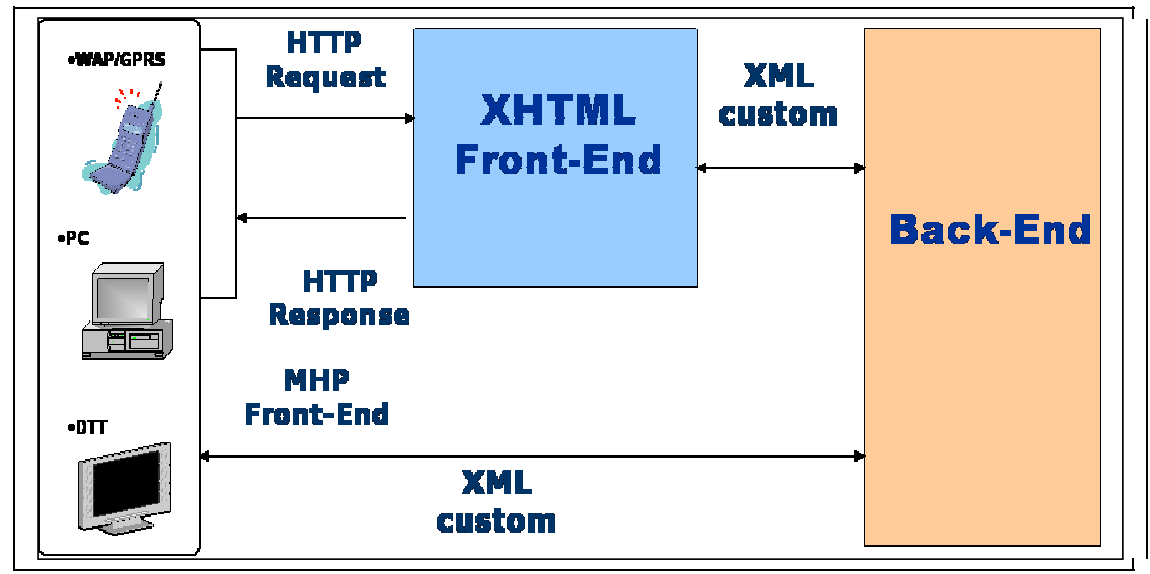

Fig. 1. The Talea architecture

The separation in two independent parts (Backend and Frontend), communicating through XML, was introduced to enable a flexible implementation of multichannel and multi-device access to the system. The current prototype supports two main categories of client: browser-enabled clients (Pc, PDA, XHTML-enabled mobile phones) and browser-less clients (stand-alone applications, MHP clients, ecc.). The first can exploit XHTML markup to populate interfaces, while the latter have no browser capabilites and need to extract content directly from XML 
objects. This is the reason why the Backend core is isolated and the Frontend can act as a "meta-client", manipulating XML content through XSL transformations to generate the final XHTML markup. The customization of the presentation layer is important for the final application, because it defines the layout in which the user interaction will take place. In order to achieve such a customization, the customizer has to implement a new Frontend or to modify a provided one, i.e. the XHTML Frontend by rewriting XSL transformations.

Finally, in order to guarantee the generality of the approach, Talea core modules do not make any assumption about domain-specific semantics: the business logic embedded within system modules is totally independent with respect to the specific semantics of resources or users. Moreover, one of the main goals of the design, was the one of making the implementation of the high-level operations as modular as possible. For this reason, there is a class for each high-level operation, in order to isolate as much as possible its management. In particular, the platform modules are based on the generator/performer design pattern, which greatly simplifies the task of adding new features (to extend functionality). Each Talea core functionality corresponds to an XML message format, devoted to a specific request/response type; each request type is handled by a specific Performer module. The Performer that is in charge of managing the user request is dynamically selected at run-time, on the basis of the request type.

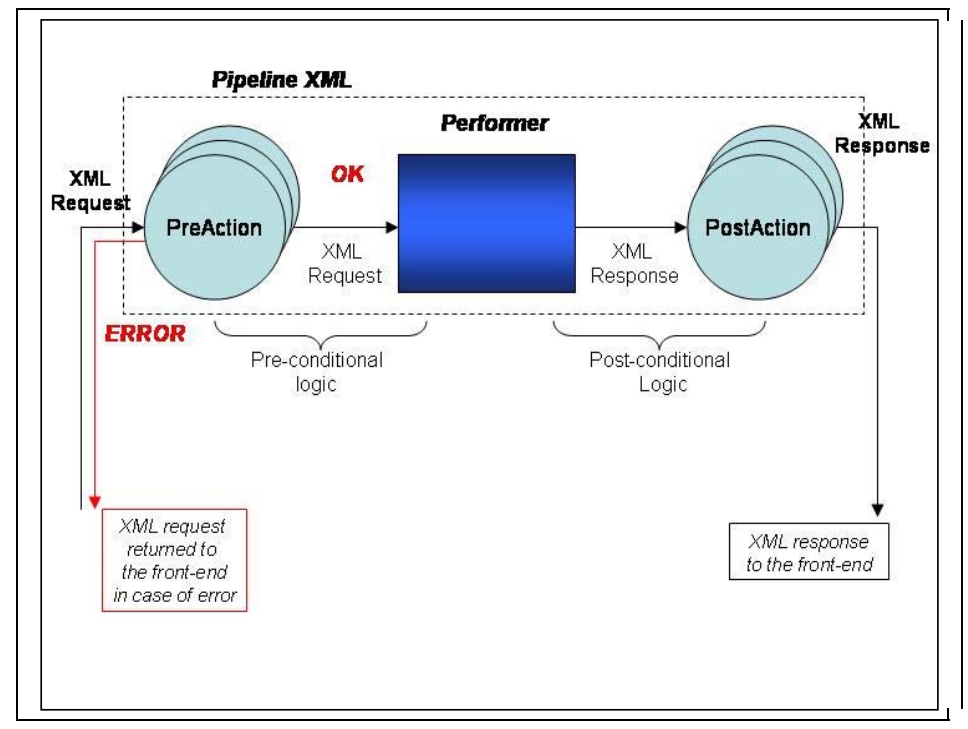

Fig. 2. Performer extension with PreAction and PostAction pipelines

The Frontend modules, based on the same pattern, manage HTTP requests and generate the corresponding XML message: for each request type (encoded in a parameter within the HTTP request), a Generator module creates the XML message (based on the HTTP request attributes), to be sent to the Backend. The interaction between Frontend and Backend follows the request/response protocol: the Frontend sends an XML message to the Backend; the Backend forwards the 
message to the specific Performer; the Performer handles the request and sends the Frontend a response message. The creation of a new Performer module enables the customizer to add high-level features to the system. For instance, in order to add new advanced search capabilities, the creation of a new Performer module that encapsulates the advanced search strategy should be required. However, the addition of a new Performer is not the only way to customize the business logic in Talea. In fact, the framework supports the modelling of domain-spcific business logics by customizing the basic Performer modules supplied with the framework, by extending their functionality when needed. To this purpose, the framework includes two public interfaces, PreAction and PostAction, that can be used to customize the Performer's pre-conditional (and post-conditional) logic. In this case, we applied some Aspect-Oriented architectural concepts, in order to enable the customizer to enrich the Performer capabilities without changing its code. As shown in Fig. 2, it is possible to attach an XML pipeline before and after the Performer execution: this facilitates the extension of the Performer's logic, without any impact on its implementation. In other words, for each Performer, it is possible to configure an arbitrary number of steps before and after its execution. The associations between each Performer and the additional modules implementing the PreAction/PostAction pipelines are stored in an XML configuration file.

\section{The Ontology}

Within the Talea framework, the ontology describes the semantics of the matchmaking process, since it represents the relations between users and services, i.e. the fact that SME provide and/or consume services. Moreover, the ontology plays a major role in the customization task, since the customizer has to describe the knowledge about the particular business domain, by providing an ontological description that includes user and service categories as well as the relations between them.

The ontology included in the Talea framework (Talea Ontology) is expressed in RDF/RDFS format [10] and represents the top-level classes: the customization process consists in the extension of this top-level. Moreover, the exploitation of a standard format such ad RDF/RDFS guarantees the possibility of exploiting standard tools, such as Protégé [9], to extend the ontology. Talea Ontology defines two classes, together with theirs properties, and the relations between them: the User class describes a generic Talea final user; the Service class describes a generic Talea service; the provide and consume relations represent the link between users and services.

The customizer can define User and Service subclasses by exploiting the Protégé editor [9]. She can also define the relations among such subclasses by specifying which Service subclasses are provided and consumed by each user category (subclass of User). For example, in the first prototype, instantiated on the

4 The User and Service classes in the Talea Ontology are generic concepts, because theirs features depends on the particular application domain. 
tourist domain, examples of subclasses of Service are Course, Excursion, Lodging; MountainCourse is a subclass of Course and MountainExcursion is a subclass of Excursion; examples of subclasses of User are MountainGuide and TravelAgency; instances of TravelAgency can consume all kind of services, while instances of MountainGuide can only provide reasources that are instances of MountainCourse and MountainExcursion (and not, for instance, of Lodging).

The application resulting from the customization of the Talea framework supports the users to provide, reserve and consume instances of the Service classes (i.e., resources). Resources are stored in a database, in order to manage the booking process, while instances of the User class (registered users) are stored in an Authentication Server in order to manage the authentication process. The structure of the resources database can be generated automatically starting from the ontology. In particular, at customization time, the customizer can select the level of the ontology classes that are converted into database tables. For instance, she can select the classes directly under Service (e.g., Course, Excursion, Lodging), or she can decide to have a larger number of smaller tables by selecting a lower level (e.g., HikingCourse, RaftingCourse, which can be subclasses of MountainCourse): an ontology class corresponding to a database table is called a table class; only table classes (and their subclasses) can have instances stored in the database. The link between each resource and its semantic description in the ontology (i.e., the class the resource belongs to) enables the Talea semantic engine to support the semantic search of services.

In the Authentication Server the information about each user includes the ontology classes she belongs to; if a user belongs to several classes at the same time (because she is, for instance, both a mountain guide and an innkeeper), she will be able to provide/consume the union of the resources that instances of those user classes are allowed to provide/consume. The link between each user and its role (i.e., the class she belongs to) supports the personalized navigation, based on the ontology structure. Let's illustrate this mechanism with an example. John, a mountain guide, connects to the system through his mobile phone in order to publish his availability for a rafting course on August $13^{\text {th }}$. After login, the system recognizes him by retrieving information from the Authentication Server: being a mountain guide, he does not consume services but provides them and, in particular, knowing the class he belongs to, the system can retrieve the type of services he can offer (e.g. MountainCourses, MountainExcursions). As a result, John will be presented a list of such classes, among which he can select the one corresponding to the resource he wants to insert; the selection action corresponds to ontology browsing (e.g., he selects MountainCourse, and then RaftingCourse). When John reaches a table class (or a subclass of a table class), he is enabled to insert a new instance of such a class or to select a more specific class going on in browsing the ontology. If we suppose that the MountainCourse is the table class, John can insert a generic mountain course or decide to insert a more specific course, e.g. a RaftingCourse (subclass of MountainCourse). When the travel agency employee searches for a RaftingCourse (or a more generic MountainCourse) available on August $13^{\text {th }}$, she will be presented a list of rafting courses, coupled with their providers; from this list she can choose the course provided by John and reserve it. 


\section{The Role of the Semantic Engine}

Talea Semantic Engine is the Backend module that performs semantic search and exploits the ontology to provide the user with personalized navigation. The Semantic Engine can be viewed as composed by different layers, as depicted in Fig. 3. In order to access the knowledge described by the ontology (in RDFS format), the Semantic Engine use SeRQL, the RDF query language supported by Sesame (see [11]). This technology was chosen after a survey of the available alternatives. A good overview can be found in [13]. In particular, the Semantic Engine exploits the set of API for RDF Schema querying and inferencing provided by Sesame for the implementation of the Talea Semantic API. The goal of the Talea Semantic API implementation is to facilitate the business logic customization by providing a set of parametric ontological queries. Such queries can be used by each Perfomer to access the semantics of resource categories (i.e. its properties, its sublcasses, and its corresponding table class), thus avoiding the direct use of SeRQL.

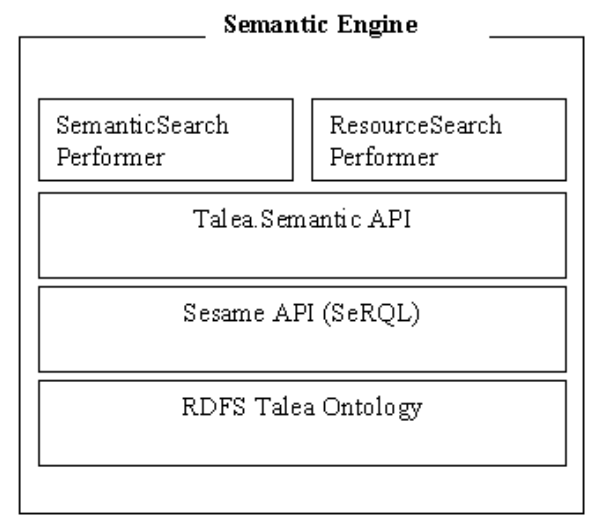

Fig. 3. The Semantic Engine

The two performers provided by the Talea framework, i.e. the Semantic Search Performer and the Resource Search Performer, use the Talea Semantic API in order to support personalized navigation and search for specific resource, respectively. The ontology-driven personalized navigation is particularly useful for devices with limited display capabilities (like smartphones or PDAs, extensively used by the users of the current prototype), since it can reduce the amount of information that the user has to provide to perform her search, because it enables her to operate only on those resources specified by the categories she belongs to.

Each user browsing action triggers a semantic search. More precisely, the request corresponding to a user action specifies the categories the user belongs to and the clicked item. The response depends on the clicked_item position in the ontology, i.e.:

- At the very beginning the user is asked to choose if she wants to insert (provide) a resource, or if she wants to look for (consume) a resource. In 
this interaction step, the user action (clicked_item) corresponds to the selection of a relation (provide or consume), thus the response is the list of service categories provided or consumed by each user_class.

- While browsing the ontology, usually the user clicks on service categories; in these cases, clicked_item is a class, thus the response is the list of its direct sublcasses.

- Finally, clicked_item can be a leaf (a class with no subclasses); in this case the response is the list of its properties; the user can express requirements about the values of such properties, that will be used to query the resources database.

In this way the Semantic Search Performer supports the navigation by looking for the "meaning" of the clicked item and providing the user with the next navigation step, on the basis of such a meaning.

The instance search into the database is performed by the Resource Search Performer: the request corresponding to a resource search is translated by the Performer in a SQL query to the database. When a user performs a resource search, i.e. she looks for instances of a particular class, the salient properties of such instances are returned by the Semantic Search Performer; in this way the user can set a list of conditions on the values of those properties (e.g., type of resource, date and time, and so on), by filling in a form. The resource search response is a list of instances that match the conditions list. The resource search/insertion functionality can be used independently from the personalized navigation and also in its place, when the ontology structure is too complex to support a user friendly navigation.

\section{Implementation Details}

Talea is implemented in Java and exploits the Java Servlet technology, running on an Apache Tomcat Web Server. The resources databases is implemented in MySQL and exploits the JDBC Connector, while the users data are stored in an LDAP Server (OpenLDAP). The genaration of user interfaces for browser-enabled devices are based on XML/XSLT tecnologies, while a MHP client has been developed for the DTT user interface. Apache Cocoon has been used as server-side web development tool. Moreover, the system relies on the already mentioned Protégé [9] and Sesame [11], the former as ontology editor and the latter to support the semantic engine. Eclipse has been used as development environment.

\section{Conclusions and Future Work}

In this paper we have introduced the first release of the Talea system that represents a starting point for the implementation of an ontology-based framework supporting e-business applications development. Talea is exploited by SME as a tool that supports the integration with legacy systems. Usually, SME already use DBMS and/or ERP for management of their resources: the Talea application is not 
aimed to substitute those systems, but rather to provide them with a middleware that enables their interoperability by means of an semantic matchmaking process.

Future works will follow two directions. First, we are going to support the selected SME during their customization process. We expect that this process will suggest us a set of improvements in the direction of a more general and powerful framework, usable in real application domains. The second direction is the implementation of a new release involving a Web Service architecture and semantic orchestration: the idea is to wrap the Performer modules in Web Services interfaces and to exploit semantic orchestration in order to provide macrofunctionality [2], [6]. We are also planning to introduce a semantic repository for instances storage. This solution would support resource semantic search (no more SQL-like), but requires the customizer to use gateway API to manage the interaction with existing DBMS or ERP.

\section{References}

[1] Alonso G., Casati F., Kuno H., Machiraju V.(2004) Web Services - Concepts, architectures and applications, Springer

[2] Bruijn J., Fensel D., Keller U., and Lara R. (2005) Using the Web Services Modelling Ontology to Enable Semantic eBusiness. Communications of the ACM (CACM), Special Issue on Semantic eBusiness

[3] Castano S., Ferrara A., Montanelli S. (2005) Dynamic Configurability of a Semantic Matchmaker for Ontology-based Resource Discovery in Open Distributed System. Proc. of INTEROP-EMOI 2005, Porto, Portugal

[4] Diadi 2000 Project: www.diadi.it/

[5] Kawamura T., DeBlasio J.A., Hasegawa T., Paolucci M., Sycara K. (2004) Public Deployment of Semantic Service Matchmaker with UDDI Business Registry. Proc. of ISWC 2004, Spinger

[6] McIlraith S. and Martin D. (2003) Bringing Semantics to Web Services. IEEE Intelligent Systems, 18(1)

[7] Paolucci M., Soudry J., Srinivasan N., Sycara K. (2005) A Broker for OWL-S Web Services. In L. Cavedon, Z. Maamar, D. Martin, and B. Benatallah (eds), Extending Web Services Technologies: the use of Multi-Agent Approaches, Kluwer

[8] Paolucci M., Sycara K., Nishimura T., Srinivasan N. (2003) Toward a Semantic Web e-commerce. Proc. of BIS 2003, Colorado Springs, USA, Abramowicz-Klein

[9] Protégé: An Ontology Editor and Knowledge-Base Framework: protege.stanford.edu/

[10] Resource Description Framework (RDF): www.w3.org/RDF/

[11] Sesame: an Open Source RDF Database with Support for RDF Schema Inferencing and Querying: www.openrdf.org

[12] Talea System: talea.csp.it/

[13] Trastour D., Bartolini C., Gonzales-Castillo, J. (2001) A Semantic Web Approach to Service Description of Matchmaking of Services. Proc. of SWW S2001, Stanford, California

[14] Trastour D., Bartolini C., Preist C. (2003) Semantic Web Support for the Business-toBusiness E-Commerce Pre-Contractual Lifecycle. Computer Networks: The Int. Journal of Computer and Telecommunications Networking, 42(5), Special Issue on The Semantic Web: an Evolution for a Revolution, North Holland/Elsevier

[15] Wrighston A. (2004) Enterprise Interoperability Architecture using XML industry Standards: www.idealliance.org/papers/dx_xmle04/papers/03-06-04/03-06-04.pdf 